\title{
Avaliação da eficácia da dieta DASH em pacientes hipertensos
}

\author{
Evaluation of the effectiveness of the DASH diet in hypertensive patients \\ Evaluación de la eficácia de la dieta DASH em pacientes hipertensos
}

Recebido: 08/02/2022 | Revisado: 14/02/2022 | Aceito: 19/02/2022 | Publicado: 01/03/2022

\author{
Júlia Galbiati de Souza \\ ORCID: https://orcid.org/0000-0003-1096-1027 \\ Universidade de São Paulo, Brasil \\ E-mail: juliagalbiati@usp.br \\ Sheilla de Oliveira Faria \\ ORCID: https://orcid.org/0000-0002-6426-932X \\ Faculdade de Medicina de São Paulo, Brasil \\ E-mail: shefaria@hotmail.com \\ Fernanda de Carvalho Vidigal \\ ORCID: https://orcid.org/0000-0001-8187-0603 \\ Universidade Federal de Alfenas, Brasil \\ E-mail: fernanda.vidigal@unifal-mg.edu.br
}

\begin{abstract}
Resumo
Objetivos: Avaliar a eficácia da dieta DASH em pacientes hipertensos em um município do sul de Minas Gerais, Brasil. Metodologia: Estudo longitudinal prospectivo, realizado com 13 pacientes hipertensos, idade média de 62,5 $\pm 10,5$ anos, recrutados de uma Clínica de Fisioterapia da cidade Alfenas, Minas Gerais. Foram realizados atendimentos individuais e em grupo. As consultas individuais ocorreram no tempo 0 (baseline), $7^{\circ}$ dia e $37^{\circ}$ após a primeira consulta. Já as atividades em grupo aconteceram no $15^{\circ}$ e $30^{\circ}$ dia após a primeira consulta. Os desfechos foram: mudanças no estado nutricional (avaliação antropométrica e da composição corporal), no padrão alimentar (score total da dieta DASH) e na PA. Resultados e Discussão: Não houve diferenças estatísticas nos desfechos estado nutricional, padrão alimentar e PA (p $>0,05)$. No entanto, os pacientes perderam em média $1,01 \pm 2,3 \mathrm{~kg}$ de peso após o $37^{\circ}$ dia de intervenção $(\mathrm{p}=0,07)$. Observou-se alta adesão da intervenção, por meio da dieta DASH, em 61,5\% pacientes $(\mathrm{n}=6)$. Conclusões: Mesmo com um pequeno tempo de intervenção (30 dias) foram observados resultados positivos quanto ao padrão dietético de alguns dos componentes da dieta DASH e uma tendência à redução no peso corporal.
\end{abstract}

Palavras-chave: Hipertensão arterial sistêmica (HAS); Padrões alimentares; Estado nutricional.

\begin{abstract}
Objective: To evaluate the efficacy of the DASH diet in hypertensive patients in a municipality in southern Minas Gerais, Brazil. Methodology: Prospective longitudinal study, performed with thirteen hypertensive subjects, mean age of $62.5 \pm$ 10.5 years, recruited from the Physical Therapy Clinic of the city of Alfenas, Minas Gerais. Individual and group visits were performed. Individual evaluation occurred at time 0 (baseline), 7th day and 37th after first evaluation. Group activities were performed on the 15th and 30th days after first evaluation. Changes in nutritional status (anthropometric and body composition), dietary pattern (total DASH diet score) and BP were considered as outcomes. Results and discussion: No statistical differences were found in nutritional status, dietary pattern and BP ( $p>0.05)$. However, it was found that subjects reduced on average $1.01 \pm 2.3 \mathrm{~kg}$ of body weight after 37 th day of intervention $(\mathrm{p}=0.07)$. High adherence of intervention was observed, regarding to DASH diet, in $61.5 \%$ patients $(\mathrm{n}=6)$. Conclusion: Even with a short intervention time (30 days), positive results were observed regarding dietary pattern of some of DASH diet components and a tendency to reduce body weight.
\end{abstract}

Keywords: Hypertension; Food patterns; Nutritional status.

\section{Resumen}

Objetivo: Evaluar la efectividad de la dieta DASH en pacientes hipertensos de una ciudad del sur de Minas Gerais, Brasil. Metodología: Estudio longitudinal prospective, realizado con 13 pacientes hipertensos, edad media de 62,5 $\pm 10,5$ años, reclutados em uma Clínica de Fisioterapia de la ciudad de Alfenas, Minas Gerias. Se realizaron sesiones individuales y grupales. Las consultas individuales se realizaron em el tiempo 0 (línea de base), 7 y 37 días después de la primeira consulta. Las actividades grupales tuvieron lugar los días 15 y 30 después de la primeira consulta. Los resultados fueron: cambios en el estado nutricional (evaluación antropométrica y de composición corporal), patrón dietético (puntuación total de la dieta DASH) y PA. Resultados y discusíon: No hubo diferencias estadísticas en el estado nutricional, patrón dietético y resultados de PA ( $\mathrm{p}>0,05)$. Sin embargo, los pacientes perdieron un promedio de $1,01 \pm 2,3 \mathrm{~kg}$ de peso después del día 37 de la intervención $(\mathrm{p}=0,07)$. Se observo alta adherencia a la intervención, a través de la dieta DASH, en el $61,5 \%$ de los pacientes $(n=6)$. Conclusión: Incluso con un tiempo de interveción corto (30 días) se observaron 
resultados positivos en cuanto al patrón dietético de algunos de los componentes de la dieta DASH y una tendencia a la reducción del peso corporal.

Palabras clave: Hipertensión; Patrones de alimentación; Estados nutricionales.

\section{Introdução}

A hipertensão arterial sistêmica (HAS) é uma doença crônica não transmissível (DCNT), sendo essa uma condição clínica multifatorial, a qual se caracteriza por níveis elevados e sustentados de pressão arterial (PA), associada frequentemente às alterações funcionais e, ou metabólicas em órgãos alvos com aumento do risco de eventos cardiovasculares, fatais ou não fatais (Malaquias et al., 2016). A HAS é uma das DCNT mais habitualmente tratadas no âmbito primário da saúde, sendo um fator importante e modificável de risco para doenças cardiovasculares e renais (Sayer et al., 2015).

Mundialmente, a HAS afeta em média 22\% da população com mais de 18 anos de idade (Rezende-Alves et al., 2020). No Brasil, de acordo com o estudo realizado em 2019 pela VIGITEL, em 27 cidades brasileiras, a frequência de diagnóstico médico de HAS foi de $24,5 \%$, sendo mais prevalente em mulheres $(27,3 \%)$ do que em homens (21,2\%). A frequência de diagnósticos aumentou com a idade e foi menor entre os indivíduos com maior nível de escolaridade ( 8 anos ou mais de escolaridade) (Brasil, 2020).

São diversos os fatores de risco para a HAS, como idade, sexo, cor da pele, excesso de peso, obesidade, ingestão de sal, consumo de álcool, sedentarismo, fatores socioeconômicos e genéticos (Bento et al., 2020).

O tratamento da HAS pode ser tanto medicamentoso quanto não medicamentoso, a depender da evolução e da gravidade da doença. A meta primordial deve ser a redução da morbidade e da mortalidade cardiovascular. Dessa forma, alimentação saudável, consumo controlado de sódio e álcool, ingestão de potássio e combate ao sedentarismo e ao tabagismo compõem as principais recomendações não medicamentosas para prevenção primária e controle da HA (Malaquias et al., 2016).

Dentre as intervenções nutricionais para a hipertensão arterial, destaca-se o padrão dietético Dietary Approaches to Stop Hypertension (DASH), que recomenda algumas alterações na dieta, como: preferência a alimentos com pouco gordura saturada, colesterol e gordura total; maior ingestão de frutas e hortaliças, aproximadamente oito a dez porções por dia; inclusão de duas ou três porções de laticínios desnatados ou semidesnatados por dia; preferência a alimentos integrais; ingestão de quatro a cinco porções por semana de oleaginosas, sementes e grãos; redução da adição de gorduras e sal nos alimentos; redução de produtos industrializados, molhos e caldos prontos; e diminuição do consumo de doces e bebidas com açúcar (Chiavaroli et al., 2019; Siervo et al., 2015).

Diversos estudos vêm demonstrando a efetividade da adoção do padrão dietético DASH no controle da HAS (Appel, 2017; Juraschek et al., 2017; Sayer et al., 2015).

Assim, o uso do padrão dietético DASH vem despertando a atenção de outros pesquisadores. No entanto, poucos estudos sobre a efetividade do padrão de dieta DASH foram realizados no Brasil. O objetivo do presente estudo foi avaliar a eficácia da dieta DASH em pacientes hipertensos em um município no sul de Minas Gerais, Brasil.

\section{Metodologia}

Trata-se de estudo longitudinal prospectivo, realizado entre setembro de 2016 e julho de 2017, em uma Clínica de Fisioterapia na cidade de Alfenas, sul de Minas Gerais.

Pacientes hipertensos atendidos na clínica foram encaminhados pelos alunos e/ou professores, de maneira não probabilística, por conveniência, sendo os critérios de inclusão, idade superior a 45 anos, ambos os sexos, portadores de HAS, capazes de responder o questionário, possuir disponibilidade para participar de atividades em grupo, concordar em participar do estudo e assinar o Termo de Consentimento Livre e Esclarecido (TCLE). Pacientes que já estavam em acompanhamento 
nutricional, gestantes ou lactantes foram excluídos. O estudo foi aprovado pelo Comitê de Ética em Pesquisa com Seres Humanos da UNIFAL-MG (número do parecer: 1.623.202; CAAE: 56662816.0.0000.5142).

Os atendimentos foram realizados tanto individualmente quanto em grupo. As consultas individuais ocorreram no tempo 0 (baseline), $7^{\circ}$ dia e $37^{\circ}$ após a primeira consulta. Já no $15^{\circ}$ e $30^{\circ}$ dia após o primeiro encontro, aconteceram as atividades em grupo.

No primeiro encontro (baseline), aplicou-se um questionário contendo informações como, perfil socioeconômico, escolaridade, hábitos de vida (consumo de bebida alcoólica, tabagismo, prática de atividade física), uso de medicamentos, antecedentes familiares e comorbidades associadas. Ademais, foram coletados no tempo 0 e no $37^{\circ}$ dia de intervenção o recordatório alimentar de 24 horas, as medidas antropométricas e aferida a PA.

A intervenção nutricional, realizada de modo individual, teve como base o padrão dietético DASH (Quadro 1) (Lichtenstein et al., 2006).

Quadro 1. Grupos dos alimentos e quantidades recomendadas em uma dieta de $2100 \mathrm{kcal}$ por dia conforme proposta original da dieta DASH.

\begin{tabular}{|l|c|}
\hline \multicolumn{1}{|c|}{ GRUPO DE ALIMENTOS } & QUANTIDADE/PORÇÕES \\
\hline Frutas (porções/dia) & $4-5$ \\
\hline Vegetais (porções/dia) & $4-5$ \\
\hline Leite e derivados <1\% de gordura (porções/dia) & $2-3$ \\
\hline Carnes magras, peixes e frangos (gramas/dia) & $<180$ \\
\hline Óleos e gorduras (porções/dia) & $2-3$ \\
\hline Sementes e oleaginosas (porções/semana) & $4-5$ \\
\hline Açúcares (porços/semana) & $<5$ \\
\hline Sal (porções/dia) & $\sim 6 \mathrm{~g}(3000 \mathrm{mg}$ de sódio) \\
\hline Grãos integrais (porções/dia) & $6-8$ \\
\hline
\end{tabular}

Fonte: Lichtenstein et al. (2006).

No $7^{\circ}$ dia após a primeira consulta, foi entregue, impresso, ao paciente o plano alimentar individualizado, juntamente com as orientações nutricionais e a lista de substituição dos alimentos.

As oficinas em grupo foram realizadas quinze dias após a primeira consulta e por volta do $30^{\circ}$ dia de intervenção, com participação ativa dos pacientes por meio de educação nutricional lúdica e prática. A primeira atividade contou com a abordagem dos "Dez Passos para uma Alimentação Adequada e Saudável”, segundo o Guia Alimentar para a População Brasileira (2014). Já na segunda atividade foram apresentadas receitas culinárias recomendadas para hipertensos em reeducação alimentar.

Foram considerados como desfechos as análises do estado nutricional (avaliação antropométrica e composição corporal), do padrão alimentar (score da dieta DASH) e da pressão arterial. Todos foram avaliados no baseline e $37^{\circ}$ após a primeira consulta.

Quanto às medidas antropométricas foram aferidos peso, estatura, perímetro do braço (PB), perímetro da cintura (PC), perímetro do quadril (PQ) e prega cutânea tricipital (PCT), seguindo as técnicas de aferição da Organização Mundial da Saúde (WHO, 1995).

Utilizou-se os pontos de corte propostos pela Sociedade Brasileira de Cardiologia (SBC) para classificar os riscos para doenças coronarianas com base no PC (ABESO, 2016).

A classificação do estado nutricional, de acordo com a PCT, segundo o percentual de adequação, foi avaliada utilizandose os critérios adaptados de Blackburn \& Thornton (1979).

A partir das medidas antropométricas calcularam-se índices antropométricos, como: índice de massa corporal (IMC), calculado a partir do peso corporal $(\mathrm{kg})$ dividido pela altura $(\mathrm{m})$ elevada ao quadrado; relação cintura/quadril (RCQ), obtida por 
meio do quociente entre as medidas de PC $(\mathrm{cm})$ e PQ $(\mathrm{cm})$; relação cintura/estatura, obtida pelo quociente entre as medidas de PC (cm) e estatura (cm) (WHO, 1995).

Os pontos de corte utilizados foram para RCQ $>0,9$ (sexo masculino) e $>0,85$ (sexo feminino) e, para RCE $>0,5$ para ambos os sexos, assim, classificando risco de desenvolver complicações metabólicas associadas à obesidade.

Para avaliar o padrão alimentar no baseline e no $37^{\circ}$ após a primeira consulta, foi aplicado o recordatório de 24 horas. A partir da avaliação, calculou-se o score da dieta DASH (Quadro 2). Para cada componente da dieta atribuiu-se um dos seguintes valores: 0 pontos (ingestão abaixo do limite inferior recomendado); 0,5 pontos (ingestão intermediária) e 1 pontos (ingestão da quantidade recomendada). Considerou-se baixa adesão à dieta uma pontuação $<6$ pontos, e alta adesão $\geq 6$ pontos.

Quadro 2. Distribuição do score da dieta DASH.

\begin{tabular}{|lccc|}
\hline \multicolumn{1}{|c}{ COMPONENTES DIETA DASH } & \multicolumn{1}{c|}{ SCORE } & $\mathbf{0}$ \\
Grãos totais & $\mathbf{1}$ & $\mathbf{0 , 5}$ & $<5$ porções/dia \\
Grãos integrais & $\geq 7$ porções/dia & $5-6$ porções/dia & $<1$ porção/dia \\
Vegetais & $\geq 2$ porções/dia & 1 porção/dia & $<2$ porções/dia \\
Frutas & $\geq 4$ porções/dia & $2-3$ porções/dia & $<2$ porções/dia \\
Laticínios desnatados & $\geq 4$ porções/dia & $2-3$ porções/dia & $<1$ porção/dia \\
Nozes, sementes e leguminosas & $\geq 2$ porções/dia & 1 porção/dia & $<2$ porções/dia \\
Carnes, aves e peixes & $\geq 4$ porções/dia & $2-3$ porções/dia & $\geq 4$ porções/dia \\
\% calorias provenientes de gordura & $\leq 2$ porções/dia & 3 porções/dia & $\geq 33 \%$ \\
$\%$ calorias provenientes de gordura saturada & $\leq 30 \%$ & $31-32 \%$ & $\geq 13 \%$ \\
Doces & $\leq 10 \%$ & $11-12 \%$ & $\geq 8$ porções/semana \\
Sódio & $\leq 5$ porções/semal & $6-7$ porções/semana & $\geq 2401 \mathrm{mg} /$ dia \\
\hline
\end{tabular}

Fonte: Folsom et al. (2007).

Ademais, de acordo com o protocolo recomendado pela SBC, para a aferição da PA utilizou-se esfigmomanômetro automático (Omron HEM-742INT).

Em relação a análise estatística, as variáveis qualitativas apresentaram-se em distribuição de frequências. Na estatística descritiva, as variáveis quantitativas com distribuição normal foram expressas em média e desvio-padrão, e em mediana (mínimo e máximo) as variáveis não paramétricas, segundo o teste Kolmogorov-Smirnov. Os desfechos, antes e após a intervenção, foram analisados a partir do teste t pareado. O nível de significância $(\alpha)$ foi de $5 \%$ e os contrastes levantados em nível bilateral. A análise estatística foi realizada com a última versão do programa SPSS para Windows.

\section{Resultados}

Avaliaram-se 13 indivíduos hipertensos, com média de idade de 62,5 $\pm 10,5$ anos, 69,2\% $(\mathrm{n}=9)$ mulheres, 61,5\% $(\mathrm{n}=8)$ casados e $84,6 \%(n=11)$ se autorreferiram como brancos. Verificou-se que $23,1 \%(n=3)$ possuíam $1^{\circ}$ grau incompleto, $23,1 \%$ ( $n$ $=3) 1^{\circ}$ grau completo e $23,1 \%(\mathrm{n}=3) 2^{\circ}$ grau completo. De acordo com a renda mensal familiar de todos os pacientes, detectou-se que a maioria $(30,8 \% ; n=4)$ tinha renda entre 1 a 3 salários mínimos.

Quanto às variáveis antropométricas, no tempo 0 (baseline), os pacientes pesavam em média 90,4 $\pm 26,3 \mathrm{~kg}$ e possuíam IMC de $35,0 \pm 8,9 \mathrm{~kg} / \mathrm{m}^{2}$ (Tabela 1 ). 
Tabela 1. Perfil antropométrico e pressão arterial dos pacientes hipertensos no início do estudo (baseline).

\begin{tabular}{lcc}
\hline Variáveis & Média & Desvio Padrão $( \pm)$ \\
\hline Peso $(\mathrm{kg})$ & 90,4 & 20,3 \\
$\mathrm{IMC}\left(\mathrm{kg} / \mathrm{m}^{2}\right)$ & 35,0 & 8,9 \\
Prega cutânea tricipital $(\mathrm{mm})$ & 25,4 & 11,6 \\
Perímetro do braço $(\mathrm{cm})$ & 34,5 & 5,7 \\
Perímetro da cintura $(\mathrm{cm})$ & 109,4 & 14,9 \\
Perímetro do quadril $(\mathrm{cm})$ & 115,5 & 18,7 \\
Pressão arterial sistólica $(\mathrm{mmHg})$ & 126,9 & 20,9 \\
Pressão arterial diastólica $(\mathrm{mmHg})$ & 69,2 & 21,0 \\
\hline
\end{tabular}

IMC: índice de massa corporal. Fonte: Autores.

Em relação às complicações metabólicas associadas à obesidade, 92,3\% $(\mathrm{n}=12)$ dos hipertensos apresentaram alto risco cardiovascular, conforme o PC, 84,6\% (n=11) em relação à RCQ, e 100\% (n=13) segundo a RCE.

Em relação ao histórico pessoal de doenças crônicas não transmissíveis (DCNT), 38,5\% (n = 5) relataram diabetes, 7,7\% $(n=1)$ dislipidemia, 92,3\% $(n=12)$ doença cardiovascular, 15,4\% $(n=2)$ obesidade e nenhum relatou possuir câncer. Outras doenças foram relatadas pelos participantes como, anemia, asma, bronquite, miastenia grave, artrite, cirurgia renal, pré-diabetes e disfunção na tireoide.

No que se refere ao histórico familiar de DCNT, notou-se que 30,8\% ( $\mathrm{n}=4)$ relataram possuir familiares com diabetes, 7,7\% (n=1) dislipidemia, 15,4\% (n=2) câncer, 69,2\% (n=9) doença cardiovascular e 30,8\% (n=4) obesidade.

Todos os pacientes faziam uso de medicamentos (média de 5,5 \pm 3,2 medicamentos), sendo 13 o número máximo de medicamentos para um mesmo paciente.

Além disso, não houve o registro de fumantes entre os atendidos, e apenas um paciente $(7,7 \%)$ relatou ter fumado há 30 anos. Já quanto ao consumo de bebidas alcoólicas, 30,8\% $(\mathrm{n}=4)$ disseram ingeri-las aos finais de semana.

A maioria dos participantes disseram praticar atividade física $(92,3 \%, \mathrm{n}=12)$, sendo 46,2\% $(\mathrm{n}=6)$ diário, 46,2\% $(\mathrm{n}=6)$ semanal, e 7,7\% ( $n=1)$ não soube responder. O tempo médio de duração das atividades era de $45 \pm 20$ minutos, sendo 20 e 90 minutos, mínimo e máximo, respectivamente. A atividade física mais praticada era a caminhada na esteira $(53,8 \% ; \mathrm{n}=7)$.

Quanto ao estado nutricional dos pacientes, no tempo baseline, foi possível analisar, segundo a classificação do IMC, que $30,8 \%(n=4)$ apresentavam sobrepeso e $61,6 \%$ obesidade $(n=8)$, sendo que nenhum dos hipertensos encontrava-se eutrófico.

Segundo a adequação da PCT, pode-se perceber que dentre os pacientes $23,1 \%(\mathrm{n}=3)$ foram classificados como desnutridos, 15,4\% $(\mathrm{n}=2)$ eutróficos $(\mathrm{PCT} 90-110 \%)$ e 23,1\% $(\mathrm{n}=3)$ obesos $(\mathrm{PCT}>120 \%)$. Analisando a adequação do PB, observa-se que 30,8\% ( $\mathrm{n}=4)$ eram eutróficos $(\mathrm{PB} 90-110 \%), 15,4 \%(\mathrm{n}=2)$ sobrepesos $(\mathrm{PB} 110-120 \%)$ e 38,5\% $(\mathrm{n}=5)$ obesos $(\mathrm{PB}>120 \%)$.

As médias das variáveis antropométricas e da pressão arterial, comparando o $37^{\circ}$ dia após a primeira consulta e o baseline, não apresentaram diferença estatística. No entanto, vale ressaltar que os pacientes perderam em média $1,1 \pm 2,3 \mathrm{~kg}$ após o período de 30 dias de intervenção (Tabela 2). 
Tabela 2. Média da diferença entre o $37^{\circ}$ dia e o tempo 0 (baseline) e desvio padrão das variáveis antropométricas e da pressão arterial.

\begin{tabular}{lccc}
\hline Variáveis antropométricas e pressão arterial & $\begin{array}{c}\text { Média da diferença } \\
\left(\mathbf{3 7}^{\mathbf{0}} \text { dia }- \text { baseline }\right)\end{array}$ & Desvio padrão & p \\
\hline Peso $(\mathrm{kg})$ & $-1,1$ & $\pm 2,3$ & 0,115 \\
IMC $\left(\mathrm{kg} / \mathrm{m}^{2}\right)$ & $-0,5$ & $\pm 1,0$ & 0,121 \\
PCT $(\mathrm{mm})$ & $-0,2$ & $\pm 2,2$ & 0,802 \\
PC $(\mathrm{cm})$ & 0,9 & $\pm 4,0$ & 0,416 \\
PB $(\mathrm{cm})$ & 0,2 & $\pm 0,8$ & 0,482 \\
RCQ & 0,01 & $\pm 0,31$ & 0,311 \\
PAS & $-3,8$ & $\pm 10,4$ & 0,209 \\
PAD & 7,7 & $\pm 29,5$ & 0,365 \\
\hline
\end{tabular}

IMC: índice de massa corporal; PCT: prega cutânea tricipital; PC: perímetro da cintura; PB: perímetro do braço; RCQ: relação cintura/quadril; PAS: pressão arterial sistólica; PAD: pressão arterial diastólica. Fonte: Autores.

Observou-se que 61,5\% $(\mathrm{n}=8)$ obtiveram uma alta adesão $(\geq 6$ pontos $)$ ao padrão alimentar da dieta DASH prescrita aos pacientes. De acordo com a Tabela 3, verifica-se que, após o período de intervenção, os pacientes portadores de HAS aumentaram o consumo dos grupos alimentares grão integral, vegetal, fruta e laticínios. Em contrapartida, reduziram o consumo de grão total. Detectou-se que o score da dieta DASH, após o período de intervenção, aumentou, apresentando uma média da diferença de $1,0 \pm$ 1,9. No entanto, não foi verificada diferença estatística $(\mathrm{p}>0,05)$ (Tabela 3).

Tabela 3. Média da diferença e desvio padrão entre o $37^{\circ}$ dia e tempo 0 (baseline) dos grupos de alimentos e do padrão alimentar dieta DASH.

\begin{tabular}{lccc}
\hline Grupo de alimento & $\begin{array}{c}\text { Média da diferença } \\
\left(\mathbf{3 7}^{\mathbf{0}} \mathbf{\text { dia }} \text { - } \text { baseline }\right)\end{array}$ & Desvio padrão & Nível de significância (p) \\
\hline Grão total & $-2,2$ & $\pm 2,2$ & $\mathbf{0 , 0 0 5}$ \\
Grão integral & 0,7 & $\pm 0,9$ & $\mathbf{0 , 0 1 3}$ \\
Vegetal & 1,5 & $\pm 1,4$ & $\mathbf{0 , 0 0 3}$ \\
Fruta & 1,0 & $\pm 1,2$ & $\mathbf{0 , 0 1 6}$ \\
Laticínios & 0,5 & $\pm 0,9$ & $\mathbf{0 , 0 4 7}$ \\
Carnes & $-1,5$ & $\pm 0,8$ & 0,502 \\
Gordura total & $-3,7$ & $\pm 9,1$ & 0,170 \\
Gordura saturada & $-2,9$ & $\pm 5,2$ & 0,065 \\
Doces & $-0,5$ & $\pm 1,6$ & 0,319 \\
Sódio & $-268,5$ & $\pm 647,2$ & 0,161 \\
& $\mathbf{1 , 0}$ & $\pm \mathbf{1 , 9}$ & $\mathbf{0 , 0 7 2}$ \\
\hline
\end{tabular}

Fonte: Autores.

\section{Discussão}

O presente estudo avaliou a eficácia da dieta DASH em pacientes hipertensos em um município no sul de Minas Gerais, Brasil. Segundo a Pesquisa de Vigilância de Fatores de Risco e Proteção para Doenças Crônicas por Inquérito Telefônico (VIGITEL), realizada em 2019, pelo Ministério da Saúde em todas as capitais do país, dentre os entrevistados, maior parte dos hipertensos eram do sexo feminino e com idade acima de 65 anos (Brasil, 2020). Segundo a Pesquisa Nacional de Saúde (PNS), a prevalência de indivíduos hipertensos foi de 21,4, sendo maior em pessoas com idade avançada, baixa escolaridade, sexo feminino e raça/cor negra (Lobo et al., 2017). Em concordância com esses achados, pode-se verificar que dentre os participantes do presente 
trabalho, a maioria eram mulheres, com escolaridade incompleta e idade superior a 65 anos. Em contrapartida, grande parte dos indivíduos eram brancos e com renda intermediária (1 a 3 salários mínimos).

No que diz respeito à composição corporal, no início do estudo foi possível observar a prevalência de participantes obesos e com perímetros (braço, cintura e quadril) aumentados. Estima-se que 20 a $30 \%$ da prevalência de HAS associa-se com o excesso de peso, e com alguns fatores de risco, como tabagismo, ingestão de bebidas alcoólicas, sedentarismo, para o desenvolvimento dessa enfermidade (Glenn et al., 2021; Nascimento et al., 2021).

Quanto à PCT, a maioria dos pacientes apresentaram-se desnutridos ou eutróficos, o que pode ser explicado pelo fato de os níveis pressóricos estarem mais interligados à obesidade central e, não tanto com a adiposidade corporal geral. O excesso de gordura na região abdominal relaciona-se com o desenvolvimento de doenças cardiovasculares (Farhadnejad et al., 2019).

A maioria dos pacientes relataram ser portadores não apenas de HAS, como também de outras comorbidades, como diabetes, dislipidemia e até mesmo outra enfermidade cardiovascular. Essas, por suas vezes, acometem, com uma maior chance indivíduos em idade avançada e com obesidade central e/ou adiposidade abdominal (Hamer \& Stamatakis, 2012).

A ingestão de medicamentos anti-hipertensivos e a prática regular de atividade física podem ser razões pelas quais a PA dos participantes encontrava-se dentro da média. A maioria dos pacientes atendidos apresentava estilo de vida aparentemente saudável.

No Brasil, é comum o uso disseminado de medicamentos, principalmente, entre a população idosa (idade $\geq 60$ anos). Tal dado vai ao encontro do presente estudo, onde nota-se que todos os participantes usavam algum medicamento (Shen et al., 2017).

No que se refere às medidas antropométricas após o tempo de intervenção (entre o $37^{\circ}$ dia e o baseline), nota-se que os pacientes reduziram algumas medidas, dentre elas peso, IMC, PCT, além da pressão arterial sistólica (PAS). No entanto, as variáveis PC, PB, RCQ e pressão arterial diastólica (PAD) apresentaram aumento das suas medidas. Por outro lado, não houve significância estatística em nenhuma dessas alterações. Determinado fato explica-se, provavelmente, devido ao curto período de intervenção. Por sua vez, se os pacientes perderam em média $1 \mathrm{~kg}$ por mês, pode-se predizer que em um período de 4 meses poderiam perder em torno de 4 a $5 \mathrm{~kg}$, o que representaria cerca de $5 \%$ de perda do peso corporal. A perda de $5 \%$ do peso corporal já é capaz de efetuar benefícios significativos ao paciente, como a redução da PA (Ponce-Martínez et al., 2020).

Com relação à dieta DASH é possível averiguar que nem todos se adequaram ao planejamento alimentar sugerido, porém, a maioria apresentou alta adesão ao plano dietético. A adesão à dieta DASH pode aumentar a ingestão de potássio, magnésio, cálcio e fibras, sendo esses contribuintes para diminuição dos níveis pressóricos (Appel, 2017).

Vale ressaltar que alguns alimentos que compõem o padrão alimentar DASH, como por exemplo, as oleaginosas, são alimentos de alto custo, o que poderia justificar o baixo consumo dessas. Sendo assim, seria conveniente trabalhar com maior assiduidade a educação nutricional junto a esses pacientes para melhores resultados quanto à transição dos hábitos alimentares. Após o período de intervenção, os pacientes hipertensos aumentaram a ingestão dos grupos alimentares como grãos integrais, vegetais, frutas e laticínios. Estes alimentos quando consumidos em conjunto contribuem para uma alimentação mais rica em fibras, minerais, gordura mono e poli-insaturada e uma redução em gorduras total, saturada e colesterol LDL, trazendo resultados benéficos para o manejo da PA. Na dieta DASH, os minerais mais presentes são o potássio, responsável por diminuir a concentração de sódio intracelular, induzindo a queda na pressão arterial; o cálcio, o qual ajuda na regulação dos batimentos cardíacos e, reduzir, quando aumentados, os níveis de sódio; e o magnésio, capaz de inibir a contração muscular lisa, regulan do a PA a partir da sua função vasodilatadora (Appel, 2017; Sayer et al., 2015).

Este trabalho apresenta algumas limitações. Primeiro, foi utilizado o recordatório de 24 horas como ferramenta para avaliar o consumo alimentar dos pacientes. A ferramenta apresenta algumas limitações, uma vez que depende da memória do paciente e talvez não reproduza a ingestão real do paciente ao longo do período estudado. Entretanto, vale ressaltar que o recordatório de 24 horas é uma das ferramentas mais utilizadas na prática clínica pela sua simplicidade e facilidade de aplicação. 
Outra limitação do estudo, foi o tempo curto de intervenção, que pode não ter sido suficiente para verificar mudanças mais expressivas nos desfechos. Logo, resultados mais eficientes poderiam ser adquiridos, caso o período de intervenção fosse maior. Finalmente, o número pequeno de pacientes também é uma limitação do trabalho. No entanto, o estudo pode servir como piloto para a implantação de intervenções nutricionais baseados no modelo DASH para outros serviços no país que pretendem implementar medidas não medicamentosas de controle de hipertensão arterial.

\section{Conclusão}

Conclui-se que apesar do curto período de tempo da intervenção nutricional foi possível observar que, mesmo não apresentando diferença estatística, houve uma considerável perda de peso corporal no decorrer do acompanhamento atrelada às mudanças no padrão alimentar, por meio da adoção da dieta DASH. Dessa forma, ao associar uma melhor alimentação juntamente à perda de algumas medidas antropométricas, como peso, nota-se o quão essas estratégias são eficazes para controle da PA.

Dessa maneira, para trabalhos futuros associando o manejo do padrão dietético DASH como um dos fatores de tratamento para a HAS, seria interessante um maior tempo de intervenção com a dieta, além da realização também de análise bioquímica de fatores inflamatórios e lipídicos antes, durante a após o início da intervenção.

\section{Referências}

Appel, L. J. (2017). The Effects of Dietary Factors on Blood Pressure. Cardiology Clinics, 35(2), 197-212. 10.1016/j.ccl.2016.12.002

Associação Brasileira para o Estudo da Obesidade e da Síndrome Metabólica - ABESO (2016). Diretrizes Brasileiras de Obesidade. (4a ed.).

Bento, I. C., Mambrini, J., \& Peixoto, S. V. (2020). Contextual and individual factors associated with arterial hypertension among Brazilian older adults (National Health Survey - 2013). Brazilian Journal of Epidemiology, 23, e200078. 10.1590/1980-549720200078

Blackburn, G. L., \& Thornton, P. A. (1979). Nutritional assessment of the hospitalized patient. Medical Clinics of North America, 63(5), 11103-11115. $10.1016 / \mathrm{S} 0025-7125(16) 31663-7$

Brasil. Ministério da Saúde. Secretaria de Vigilância em Saúde. Departamento de Análise em Saúde e Vigilância de Doenças Não Transmissíveis. (2020). Vigitel Brasil 2019: Vigilância de fatores de risco e proteção para doenças crônicas por inquérito telefônico: estimativas sobre frequência e distribuição sociodemográfica de fatores de risco e proteção para doenças crônicas nas capitais dos 26 estados brasileiros e no Distrito Federal em 2019 [recurso eletrônico]. Brasília: Ministério da Saúde.

Chiavaroli, L., Viguiliouk, E., Nishi, S. K., Blanco Mejia, S., Rahelić, D., Kahleová, H., Salas-Salvadó, J., Kendall, C. W., \& Sievenpiper, J. L. (2019). DASH Dietary Pattern and Cardiometabolic Outcomes: An Umbrella Review of Systematic Reviews and Meta-Analyses. Nutrients, 11(2), 338. https://doi.org/10.3390/nu11020338

Farhadnejad, H., Darand, M., Teymoori, F., Asghari, G., Mirmiran, P., \& Azizi, F. (2019). The association of Dietary Approach to Stop Hypertension (DASH) diet with metabolic healthy and metabolic unhealthy obesity phenotypes. Scientific Reports, 9(1), 18690. 10.1038/s41598-019-55285-6

Folsom, A. R., Parker, E. D., \& Harnack, L. J. (2007). Degree of concordance with DASH diet guidelines and incidence of hypertension and fatal cardiovascular disease. American Journal of Hypertension, 20(3), 225-232. 10.1016/j.amjhyper.2006.09.003

Glenn, A. J., Hernández-Alonso, P., Kendall, C., Martínez-González, M. Á., Corella, D., Fitó, M., Martínez, J. A., Alonso-Gómez, Á. M., Wärnberg, J., Vioque, J., Romaguera, D., López-Miranda, J., Estruch, R., Tinahones, F. J., Lapetra, J., Serra-Majem, J. L., Bueno-Cavanillas, A., Tur, J. A., Celada, S. R., Pintó, X., ... Salas-Salvadó, J. (2021). Longitudinal changes in adherence to the portfolio and DASH dietary patterns and cardiometabolic risk factors in the PREDIMED-Plus study. Clinical nutrition (Edinburgh, Scotland), 40(5), 2825-2836. https://doi.org/10.1016/j.clnu.2021.03.016

Juraschek, S. P., Miller, E. R., Weaver, C. M., \& Appel, L. J. (2017). Effects of Sodium Reduction and the DASH Diet in Relation to Baseline Blood Pressure. Journal of the American College of Cardiology, 70(23), 2841-2848. 10.1016/j.jacc.2017.10.011

Hamer, M., \& Stamatakis, E. (2012). Metabolically healthy obesity and risk of all-cause and cardiovascular disease mortality. The Journal of Clinical Endocrinology \& Metabolism, 97(7), 2482-2488. 10.1210/jc.2011-3475

Lichtenstein, A. H., Appel, L. J., Brands, M., Carnethon, M., Daniels, S., Franch, H. A., Wylie-Rosett, J. (2006). Diet and lifestyle recommendations revision 2006: A scientific statement from the American Heart Association Nutrition Committee. Circulation, 114(1), 82-96. 10.1161/CIRCULATIONAHA.106.176158

Lobo, L., Canuto, R., Dias-da-Costa, J. S., \& Pattussi, M. P. (2017). Tendência temporal da prevalência de hipertensão arterial sistêmica no Brasil. Cadernos de Saúde Pública, 33(6), e00035316. 10.1590/0102-311X00035316

Malachias, M. V. B., Plavnik, F. L., Machado, C. A., Malta, D., Scala, L. C. N., \& Fuchs, S. (2016). $7^{\mathrm{a}}$ Diretriz Brasileira de Hipertensão Arterial: Capítulo 1 Conceituação, Epidemiologia e Prevenção Primária. Arquivos Brasileiros de Cardiologia, 107(3, Suppl. 3), 1-6. 10.5935/abc.20160151 
Research, Society and Development, v. 11, n. 3, e39811326723, 2022

(CC BY 4.0) | ISSN 2525-3409 | DOI: http://dx.doi.org/10.33448/rsd-v11i3.26723

Nascimento, R. L. do, Carvalho, F. O., Araujo, F. de S.., Melo-Marins, D. de., Carneiro, M. V. O., Saraiva, L. C., Moreira, S. R., \& Nascimento Junior, J. R. A. (2021). Indicadores antropométricos e hemodinâmicos associados à hipertensão arterial em pessoas sedentárias. Pesquisa, Sociedade $e$ Desenvolvimento, 10 (7), e25310716603. https://doi.org/10.33448/rsd-v10i7.16603

Ponce-Martínez, X., Colín-Ramírez, E., Rodríguez-Ramírez, S., Rivera-Mancía, S., Cartas-Rosado, R., \& Vallejo-Allende, M. (2022). Adherence to the DASH dietary pattern is associated with blood pressure and anthropometric indicators in Mexican adults. La adherencia al patrón alimentario DASH se relaciona con la presión arterial y los indicadores antropométricos en adultos mexicanos. Nutricion hospitalaria,39(1), 128-137. https://doi.org/10.20960/nh.03728

Rezende-Alves, K., Hermsdorff, H., Miranda, A., Lopes, A., Bressan, J., \& Pimenta, A. M. (2020). Food processing and risk of hypertension: Cohort of Universities of Minas Gerais, Brazil (CUME Project). Public Health Nutrition, 1-9. 10.1017/S1368980020002074

Sayer, R. D., Wright, A. J., Chen, N., \& Campbell, W. W. (2015). Dietary Approaches to Stop Hypertension diet retains effectiveness to reduce blood pressure when lean pork is substituted for chicken and fish as the predominant source of protein. The American Journal of Clinical Nutrition, 102(2), 302-308. 10.3945/ajcn.115.111757

Shen, Y., Chang, C., Zhang, J., Jiang, Y., Ni, B., \& Wang, Y. (2017). Prevalence and risk factors associated with hypertension and prehypertension in a working population at high altitude in China: a cross-sectional study. Environmental Health and Preventive Medicine, 22(1), 19. 10.1186/s12199-017-0634-7

Siervo, M., Lara, J., Chowdhury, S., Ashor, A., Oggioni, C., \& Mathers, J. C. (2015). Effects of the Dietary Approach to Stop Hypertension (DASH) diet on cardiovascular risk factors: a systematic review and meta-analysis. The British journal of nutrition, 113(1), 1-15. https://doi.org/10.1017/S0007114514003341

World Health Organization (1995). Physical status: The use and interpretation of anthropometry. Report of a WHO Expert Committee. WHO. 